\title{
Metabolite identification using infrared ion spectroscopy - novel biomarkers for pyridoxine-dependent epilepsy
}

Rianne E. van Outersterp ${ }^{1 \pm}$, Udo F.H. Engelke ${ }^{2 \pm}$, Jona Merx ${ }^{3 \pm}$, Giel Berden ${ }^{1}$, Mathias Paul ${ }^{4}$, Thomas Thomulka ${ }^{4}$, Albrecht Berkessel ${ }^{4}$, Marleen C.D.G. Huigen ${ }^{2}$, Leo A.J. Kluijtmans ${ }^{2}$, Jasmin Mecinović ${ }^{5}$, Floris P.J.T. Rutjes ${ }^{3}$, Clara D.M. van Karnebeek ${ }^{6}$, Ron A. Wevers ${ }^{2}$, Thomas J. Boltje ${ }^{3 *}$, Karlien L.M. Coene ${ }^{2 *}$, Jonathan Martens ${ }^{1 *}$, Jos Oomens ${ }^{1,7 *}$

${ }^{1}$ Radboud University, Institute for Molecules and Materials, FELIX Laboratory, Toernooiveld 7, 6525ED Nijmegen, The Netherlands

${ }^{2}$ Department of Laboratory Medicine, Translational Metabolic Laboratory, Radboud University Medical Center, Geert Grooteplein Zuid 10, 6525 GA, Nijmegen, Netherlands

${ }^{3}$ Radboud University, Institute for Molecules and Materials, Synthetic Organic Chemistry, Heyendaalseweg 135, 6525 AJ, Nijmegen, The Netherlands

${ }^{4}$ University of Cologne, Department of Chemistry, Greinstrasse 4, 50939 Cologne, Germany

${ }^{5}$ Department of Physics, Chemistry and Pharmacy, University of Southern Denmark, Campusvej 55, 5230, Odense, Denmark

${ }^{6}$ Department of Pediatrics-Metabolic Diseases, Radboud Center for Mitochondrial Medicine, Radboud University Medical Center, Geert Grooteplein Zuid 10, 6525 GA, Nijmegen, The Netherlands.

${ }^{7}$ van't Hoff Institute for Molecular Sciences, University of Amsterdam, 1098XH Amsterdam, Science Park 908, The Netherlands

*corresponding author

${ }^{ \pm}$These authors contributed equally

\begin{abstract}
Untargeted LC-MS based metabolomics strategies are being increasingly applied in metabolite screening for a wide variety of medical conditions. The long-standing "grand challenge" in the utilization of this approach is metabolite identification - confidently determining the chemical structures of $\mathrm{m} / \mathrm{z}$-detected unknowns. Here, we use a novel workflow based on the detection of molecular features of interest by high-throughput untargeted LC-MS analysis of patient body fluids combined with targeted molecular identification of those features using infrared ion spectroscopy (IRIS), effectively providing diagnostic IR fingerprints for mass-isolated targets. A significant advantage of this approach is that in silico predicted IR spectra of candidate chemical structures can be used to suggest the molecular structure of unknown features, thus mitigating the need for the synthesis of a broad range of physical reference standards. Pyridoxine dependent epilepsy (PDE-ALDH7A1) is an inborn error of lysine metabolism, resulting from a mutation in the ALDH7A1 gene that leads to an accumulation of toxic levels of $\alpha$-aminoadipic semialdehyde ( $\alpha$-AASA), piperideine-6-carboxylate (P6C), and pipecolic acid in body fluids. While $\alpha$-AASA and P6C are known biomarkers for PDE in urine, their instability makes them poor candidates for diagnostic analysis from blood, which would be required for application in newborn screening protocols. Here, we use combined untargeted metabolomics-IRIS to identify several new biomarkers for PDE-ALDH7A1 that can be used for diagnostic analysis in urine, plasma, and cerebrospinal fluids, and are compatible with analysis in dried blood spots for newborn screening. The identification of these novel metabolites has directly rendered novel insights in the pathophysiology of PDE-ALDH7A1 ${ }^{1}$.
\end{abstract}

\section{Conflict of interest:}

Authors declare no conflicts of interest.

Ethical approval:

The work described in this study has been carried out in accordance with The Code of Ethics of the World Medical Association (Declaration of Helsinki) for experiments involving humans. All patients (or their guardians) approved of the possible use of their anonymized left-over samples for method validation purposes, in agreement with institutional and national legislation.

Keywords: Pyridoxine-dependent epilepsy, $\alpha$-aminoadipic semialdehyde ( $\alpha$-AASA) dehydrogenase, antiquitin, metabolite identification, untargeted metabolomics, infrared ion spectroscopy, molecular structure analysis, lysine metabolism, next generation metabolic screening (NGMS). 


\section{INTRODUCTION}

The metabolomics community increasingly relies on untargeted LC-MS based metabolic screening to detect novel metabolites that can be correlated to various conditions, treatments, and environments - so called, biomarkers. What is widely acknowledged as the "grand challenge" or "bottleneck" of this approach is the identification of a metabolite's molecular structure ${ }^{2-4}$. While the detection of metabolites directly from body fluids or tissues and the identification of their elemental composition, is now routinely performed down to low nanomolar (nM) abundances, these techniques cannot directly resolve molecular structures. The most common approach to molecular identification is tandem mass spectrometry (MS/MS), which maintains the high sensitivity of the LC-MS experiment. However, many isomeric structures show nearly identical MS/MS fragmentation spectra and their interpretation, which is to date challenging to model in silico ${ }^{3,5}$, requires physical reference standards. Conversely, NMR spectroscopy is a powerful technique to identify molecular structure, however, it is limited in terms of sensitivity and cannot delve deeper into the metabolome than the micromolar $(\mu \mathrm{M})$ level; moreover, tedious purification steps are usually required prior to analysis.

Very recently, several groups have suggested infrared ion spectroscopy (IRIS) as an alternative mass spectrometrybased technique for small molecule identification ${ }^{6-15}$. By combining the sensitivity and mass-selectivity of MS(/MS) with infrared spectroscopy-based orthogonal structural characterization, IRIS is able to provide detailed structural information to annotate detected features arising from MS analyses. IRIS can provide a mass-selective fingerprint IR spectrum for essentially any ion detected in an MS experiment. These IR spectra can be matched to reference IR spectra measured from standards or predicted by quantum- chemical computations, which is now routinely done using commercially available software packages. Recently, several proof-of-principle studies have successfully demonstrated the potential for metabolite identification using this technology ${ }^{6-}$ 9, 16-17. Other studies have demonstrated the coupling of IRIS with online or offline LC and paved the way towards full integration of IRIS in untargeted metabolomics workflows ${ }^{6,8}$, 13,18 .

In this study, we use IRIS for metabolite identification in the search for novel biomarkers for the inborn error of metabolism pyridoxine-dependent epilepsy (OMIM 266100, PDEALDH7A1) ${ }^{19-20}$ (see Scheme 1). PDE is an error of lysine metabolism presenting intractable epileptic seizures in the neonatal period and known to result from pathogenic mutations in the $A L D H 7 A 1$ gene that encodes the enzyme $\alpha-$ aminoadipic semialdehyde ( $\alpha$-AASA) dehydrogenase, also known as antiquitin (ATQ). ${ }^{21}$ ATQ deficiency leads to the accumulation of $\alpha$-AASA and its imine form piperidine-6carboxylic acid (P6C) in body fluids and tissues. P6C inhibits vitamin B6 (pyridoxine) function through deactivation in a spontaneous reaction ${ }^{22-23}$.

The secondary pyridoxine deficiency that arises is thought to be pathophysiological for PDE-ALDH7A $1^{21}$, as pyridoxine is a crucial co-factor for many enzymatic processes. In most cases, treatment with high-doses of pyridoxine prevents seizures in patients. However, mild to moderate intellectual disability is still highly prevalent, especially when therapy was not started early in life due to diagnostic delay. While PDE-ALDH7A1 is a relatively common metabolic disease (estimated to occur in 1:60 000 conceptions $^{24}$ ), it likely remains underdiagnosed, as no reliable biomarkers are available for early detection of this disease in newborn screening. P6C and $\alpha$-AASA are currently used as diagnostic PDE-ALDH7A1 biomarkers in urine ${ }^{25}$. For plasma, an LCMS/MS workflow has been demonstrated for simultaneous

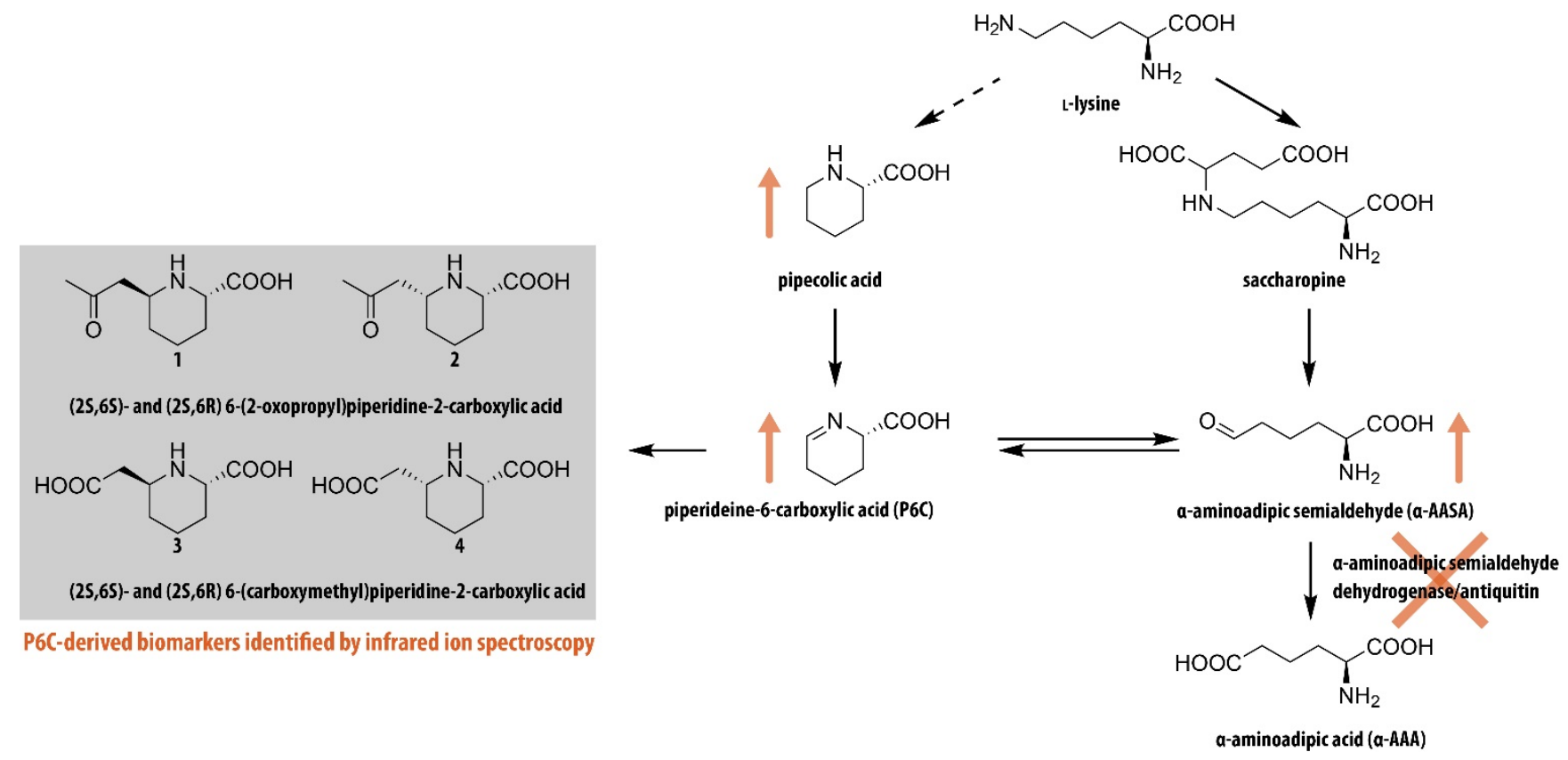

Scheme 1 | Overview of the L-lysine metabolic pathway involved in pyridoxine dependent epilepsy. Inactivity of the enzyme antiquitin coded by the ALDH7A1 gene leads to accumulation of $\alpha$-aminoadipic semialdehyde ( $\alpha$-AASA) and its cyclic counterpart piperideine-6-carboxylic acid (P6C). P6C can react spontaneously with several $\beta$-ketoacids or $\beta$-diacids resulting in the formation of a range of P6C-derived biomarkers (1-4), several of which are identified here. 
detection of P6C and $\alpha$-AASA after derivatization ${ }^{26-28}$. However, as a result of their high chemical instability, these biomarkers are not suited for application in newborn screening programs which are regularly based on direct infusion MS analysis of dried blood spots $^{29}$. Thus, there currently remains a clinical need for novel PDE-ALDH7A1 biomarkers that could be applied in newborn screening and that would enable early diagnosis and optimal clinical outcomes for patients.

In our group we have validated an untargeted metabolic profiling method based on UHPLC-MS, coined "next generation metabolic screening (NGMS)", for the diagnostic screening of inborn errors of metabolism ${ }^{30}$. Apart from diagnosis based on known biomarkers, untargeted metabolomics offers the opportunity to detect new biomarkers. However, the chemical identification of these deviating features has been the limiting factor until now. As a major step forward, we have now combined NGMS with IRIS, which covers the complete route from biomarker discovery to chemical identification. In this study, we describe the successful application of this NGMS-IRIS strategy for the start-to-finish identification of several novel biomarkers for PDE-ALDH7A1.

\section{RESULTS AND DISCUSSION}

LC-MS/MS analysis of PDE samples. We analyzed 11 plasma samples of seven genetically confirmed PDEALDH7A1 patients with bi-allelic pathogenic ALDH7A1 mutations using NGMS. We detected elevated levels of $\alpha$ AASA ( $m / z$ 146.0816, +ESI), P6C ( $\mathrm{m} / \mathrm{z}$ 128.0706, +ESI) and pipecolic acid $(\mathrm{m} / \mathrm{z} 130.0816,+E S I)$, in line with what is reported in the literature (see Table 1$)^{31}$. Apart from these metabolites, we observed several $\mathrm{m} / \mathrm{z}$ features that were previously unreported. Two features, here labelled as A and $\mathbf{B}$ both having an $m / z$ value of 186.1123 (+ESI) and retention times of 2.33 and 2.55 minutes, respectively, were noted to be significantly increased in comparison to controls. This was also the case in urine and cerebrospinal fluid (CSF) of PDEALDH7A1 patients (Table 1). Figure 1a plots the summed intensity of feature $\mathbf{A}$ and $\mathbf{B}$ in the plasma of 26 controls (grey) and 10 PDE patients (orange) measured in duplicate. Intensity plots of feature $\mathbf{A}$ and $\mathbf{B}$ are shown in Figure S1. The average fold changes (PDE-ALDH7A1 patient/control) of features A and $\mathbf{B}$ are determined at 145 and 200, respectively,
Table 1 | Overview of metabolites identified by NGMS to correlate with PDE.

\section{Compound $m / z \quad R T(m i n) \quad$ CSF Plasma Urine}

\begin{tabular}{l|llllll}
\hline A & 186.1123 & 2.33 & + & + & + \\
B & 186.1123 & 2.55 & + & + & + \\
$\mathbf{C}$ & 188.0919 & 1.45 & + & + & + \\
D & 188.0919 & 1.49 & + & + & + \\
6-oxoPIP & 144.0652 & 3.05 & + & + & + \\
a-ASAA & 146.0816 & 0.86 & + & + & + \\
P6C & 128.0706 & 0.86 & + & + & +
\end{tabular}

enabling the distinction of patients from the control group in all cases. A molecular formula of $\left[\mathrm{C}_{9} \mathrm{H}_{15} \mathrm{NO}_{3}+\mathrm{H}\right]^{+}(\Delta m=$ $-3.9 \mathrm{ppm}$ ) was assigned to these features, however neither the HMDB $^{32-35}$, METLIN ${ }^{36}$, nor ChemSpider ${ }^{37}$ libraries contained entries for this chemical formula that would be of biochemical relevance to PDE-ALDH7A1.

To resolve the molecular structures of feature $\mathbf{A}$ and $\mathbf{B}$, MS/MS spectra were recorded using collision induced dissociation (CID), see Figure $1 \mathrm{~b}$ for the MS/MS spectrum of feature A. Dissociation primarily leads to loss of $\mathrm{CO}+\mathrm{H}_{2} \mathrm{O}$ $(\Delta m=1.7 \mathrm{ppm})$ and $\mathrm{C}_{3} \mathrm{H}_{6} \mathrm{O}(\Delta m=4.6 \mathrm{ppm})$, giving fragments with $\mathrm{m} / \mathrm{z} 140.1069$ and $\mathrm{m} / \mathrm{z} 128.0707$, respectively. Moreover, the $\mathrm{m} / \mathrm{z} 128.0707$ fragment shows secondary fragmentation to $\mathrm{m} / \mathrm{z} 100.0768, \mathrm{~m} / \mathrm{z} 82.0652$ and $\mathrm{m} / \mathrm{z} 55.0542$ which can be assigned as loss of $\mathrm{CO}(\Delta m=-36 \mathrm{ppm}), \mathrm{CO}+\mathrm{H}_{2} \mathrm{O}(\Delta m=-0.5$ ppm) and $\mathrm{CO}+\mathrm{H}_{2} \mathrm{O}+\mathrm{HCN}(\Delta m=-3.7 \mathrm{ppm})$, respectively. Of particular interest is the $\mathrm{m} / \mathrm{z} 128.0707$ fragment as this corresponds to the mass of $[\mathrm{P} 6 \mathrm{C}+\mathrm{H}]^{+}$, a known biomarker of PDE-ALDH7A1 (see Scheme 1 and Table 1). Fragmentation of the unknowns $\mathbf{A}$ and $\mathbf{B}$ to P6C would suggest that their molecular structures are related to P6C. Specifically, this suggests a molecular structure consisting of $\mathrm{P} 6 \mathrm{C}$ with an additional $\mathrm{C}_{3} \mathrm{H}_{5} \mathrm{O}$-group, which is lost as a neutral fragment upon CID (see the scheme in Figure 1b).

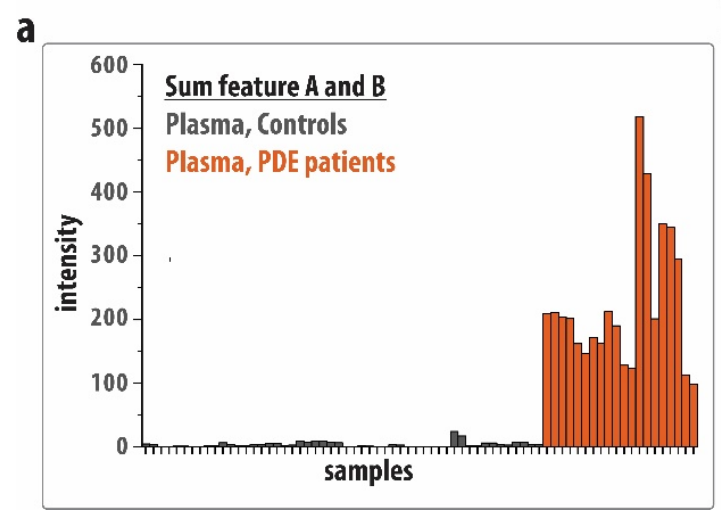

b

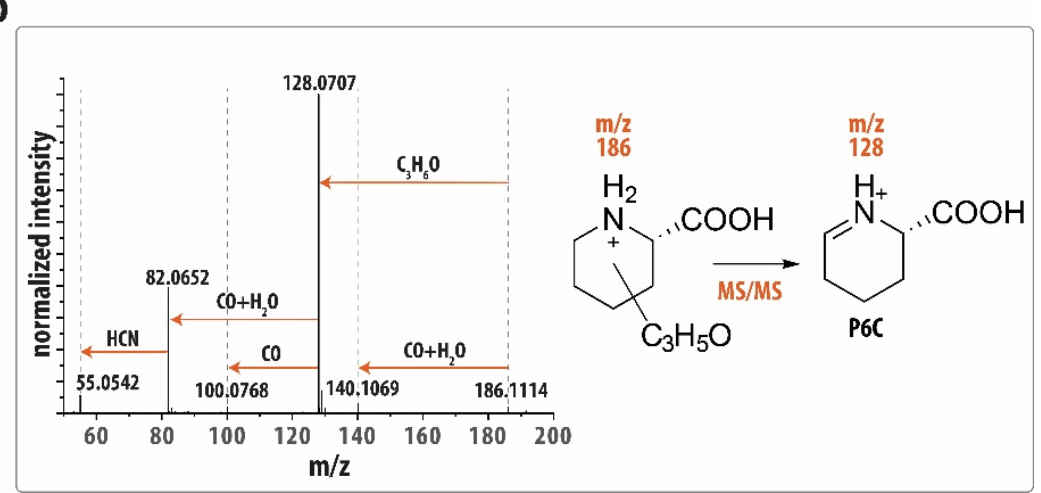

Figure 1 | Results of LC-MS/MS analysis of PDE plasma samples. (a) Plot of the sum of feature A $(\mathrm{m} / \mathrm{z} 186.1123,2.33 \mathrm{~min}$ RT) and feature $\mathbf{B}(\mathrm{m} / \mathrm{z}$ 186.1123, $2.55 \mathrm{~min}$ RT) in the plasma of 26 controls (grey) and 11 PDE patients (orange). Each sample was analyzed as two technical replicates, shown as consecutive bars in the figure. All values are normalized on the value in a quality control sample. (b) CID MS/MS spectrum of feature A in one of the PDE patient plasmas. A scheme showing the hypothesized core structure of feature $\mathbf{A}$ and $\mathbf{B}$ and their fragmentation to the $\mathrm{m} / \mathrm{z} 128.0707$ fragment is inlayed in the figure. 
Identification of the $m / z$ 128.0707 MS/MS fragment using IRIS and quantum-chemical calculations. For further identification of feature $\mathbf{A}$ and $\mathbf{B}$, we relied on IRIS and quantum-chemically predicted IR spectra. LC fractions containing either feature $\mathbf{A}$ or $\mathbf{B}$ were obtained and used to obtain IR spectra of the LC-separated, mass-isolated features as well as their CID-generated $m / z 128.0707$ fragment. Figure 2a shows the IR spectrum of the $m / z 128.0707$ fragment of feature A (black trace in both panels), which was found to be identical to the spectrum of the $\mathrm{m} / \mathrm{z} 128.0707$ fragment of feature $\mathbf{B}$ (see Figure S2). Qualitatively, one may correlate the peaks at $\sim 1800$ and $\sim 1650 \mathrm{~cm}^{-1}$ with carbonyl and $\mathrm{N}-\mathrm{H}$ functionalities, which are in agreement with the hypothesis that the fragment corresponds to protonated P6C, but other (positional) isomers cannot be directly excluded. A more confident structural assignment was derived from comparison against theoretical reference spectra of protonated P6C and a closely related isomer, protonated piperideine-2-carboxylic acid (P2C). Density functional theory (DFT) at the B3LYP/6$31++G(d, p)$ level was used to predict their vibrational spectra (orange traces in Figure 2a). The IR spectrum computed for $[\mathrm{P} 6 \mathrm{C}+\mathrm{H}]^{+}$was in excellent agreement with the experimental spectrum, whereas the computational $[\mathrm{P} 2 \mathrm{C}+\mathrm{H}]^{+}$spectrum showed clear spectral discrepancies and shifts with respect to the experimental spectrum, especially in the $1200-1500 \mathrm{~cm}^{-1}$ region. The experimental peak around $1650 \mathrm{~cm}^{-1}$ was found to be red-shifted compared to both computational spectra. We assigned this peak as an $\mathrm{N}-\mathrm{H}$ bending vibration, which are known to be inaccurately predicted by frequency calculations within the harmonic approximation due to its relatively strong anharmonic character ${ }^{38-39}$. Overall, this led us to the conclusion that we have identified the $\mathrm{m} / \mathrm{z} 128.1123$ fragment ion of both features $\mathbf{A}$ and $\mathbf{B}$ as protonated P6C.

Preliminary identification of features $A$ and $B$ using $H / D$ exchange and IRIS. Having identified the $\mathrm{C}_{3} \mathrm{H}_{6} \mathrm{O}$-loss fragment as P6C, potential structures for features $\mathbf{A}$ and $\mathbf{B}$ could be hypothesized. MS/MS ion chemistry suggests that the precursor structure likely consists of a P6C-core with an additional $\mathrm{C}_{3} \mathrm{H}_{5} \mathrm{O}$-functionalization on the ring at the location of the double bond (see scheme in Figure 1b). Figure 2b shows the IR spectra of feature $\mathbf{A}$ and $\mathbf{B}$ obtained using IRIS (black and dashed grey traces in all panels). The spectra show a high degree of similarity, which suggests that $\mathbf{A}$ and $\mathbf{B}$ possess highly-similar molecular structures and that they could be, for example, diastereomers. The major difference between the two spectra is a peak shift near $1600 \mathrm{~cm}^{-1}$ and a difference in peak shape between 1200 and $1400 \mathrm{~cm}^{-1}$. Two carbonyl a

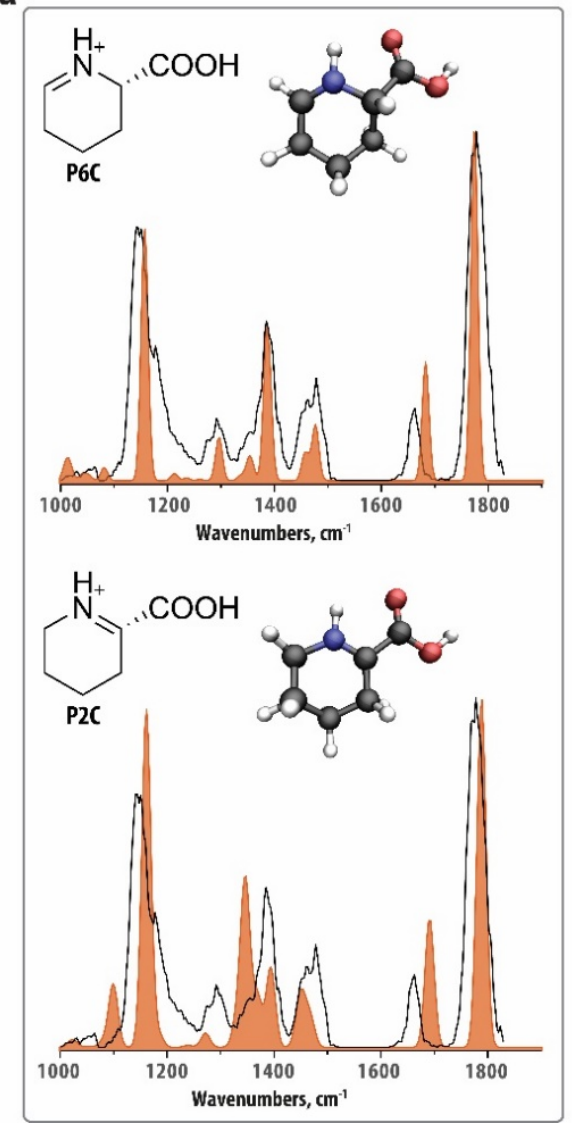

b

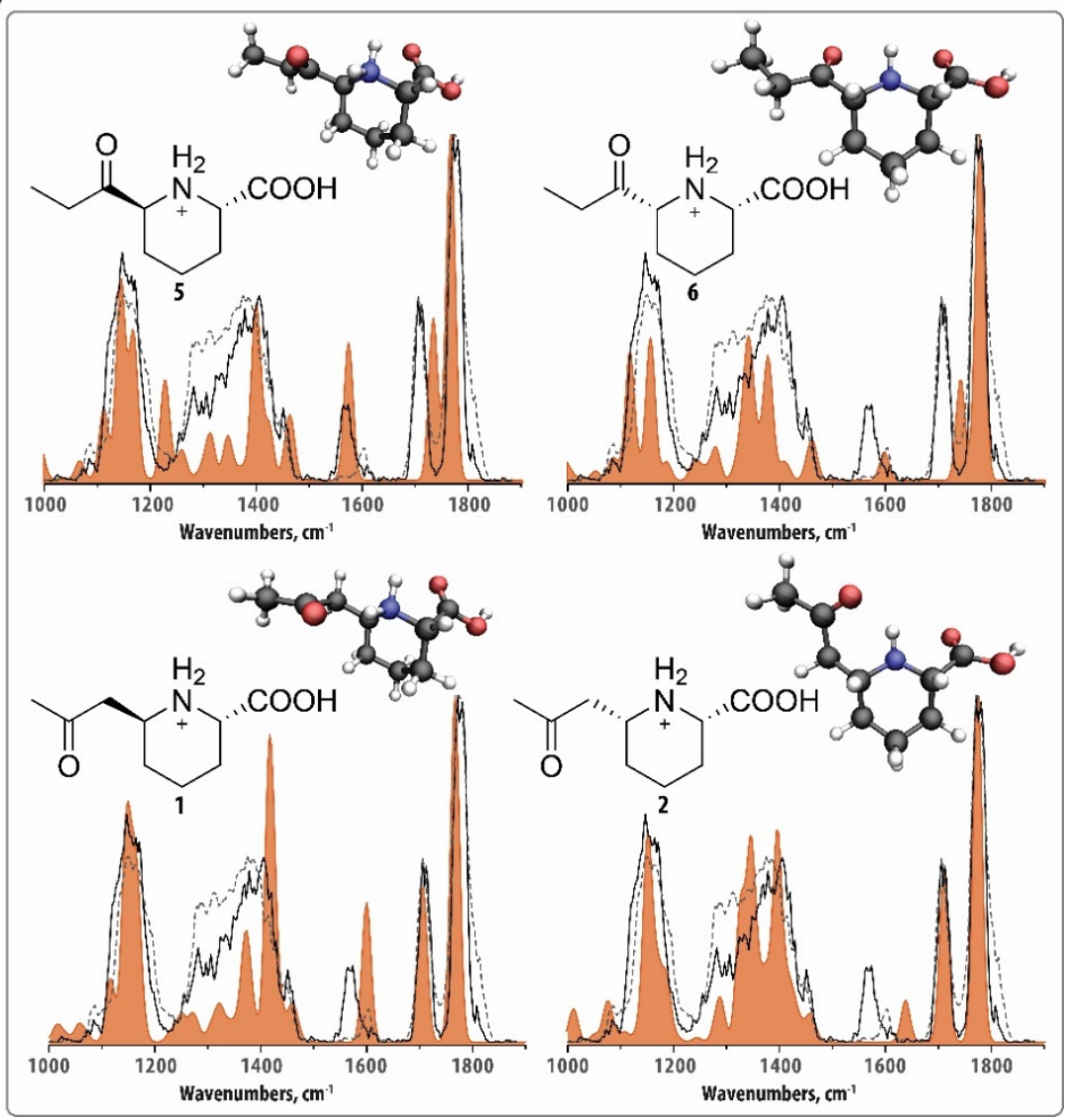

Figure 2 | Molecular identification of feature A and B and their main fragment using computational modelling and IRS. (a) Experimental IR spectrum of the $\mathrm{m} / \mathrm{z} 128.0707$ fragment of feature A (black trace in both panels) in comparison to theoretical IR spectra of protonated P6C (top panel, orange trace) and protonated 1-propanoylpiperidine-2-carboxylic acid (bottom panel, orange trace). (b) Experimental IR spectrum of feature A (black trace in all panels) and feature $\mathbf{B}$ (dashed grey trace in each panel) in comparison to theoretical IR spectra of candidate structure $\mathbf{5}$ and $\mathbf{6}$ (top left and right panel, orange trace) and candidate structure $\mathbf{1}$ and 2 (bottom left and right panel, orange trace). Input structures for the computations and the conformations resulting from the quantum-chemical calculations are inlayed in each panel. 
stretching vibrations above $1700 \mathrm{~cm}^{-1}$ are observed suggesting that both features have two $\mathrm{C}=\mathrm{O}$ groups in their structures. This suggests that, in addition to the carbonyl group of the carboxylic acid in the P6C-core, the oxygen atom in the $\mathrm{C}_{3} \mathrm{H}_{5} \mathrm{O}$-group is also present as a carbonyl.

A remaining question is whether the $\mathrm{P} 6 \mathrm{C}$-core is $\mathrm{N}$ - or $\mathrm{C}$ functionalized. To determine this, we performed H/Dexchange experiments on features $\mathbf{A}$ and $\mathbf{B}$ (see methods section for details). We observed that both features undergo a 3 amu mass shift from $\mathrm{m} / \mathrm{z} 186$ to $\mathrm{m} / \mathrm{z} 189$, indicating the presence of three rapidly exchangeable protons. The $\mathrm{C}_{3} \mathrm{H}_{5} \mathrm{O}$ group contains a carbonyl-group and thus no exchangeable protons and the carboxylic acid moiety contains one exchangeable proton. Taking into account the ionizing proton, this suggests that the ring nitrogen is a secondary amine, protonated on that nitrogen, and that our target is therefore Cfunctionalized.

Identification of feature $A$ and $B$ using quantum-chemical calculations. Based on the chemical characteristics outlined above, two plausible molecular structures can be proposed: 6(2-oxopropyl)piperidine-2-carboxylic acid (1 and 2) and 6propionylpiperidine-2-carboxylic acid (5 and 6). These molecules, shown in Figure 2b, each have two stereocenters, which result in eight possible stereo-isomers. IR spectroscopy cannot distinguish between enantiomers ( $R R$ and $S S$ ) but diastereomers ( $S S$ and $S R$ ) may show differences in their IR spectra. As the unknowns are likely reaction products of P6C, a downstream metabolite of L-lysine, we only considered the stereoisomers that were $S$ at the carboxylic acid-position.

We computed theoretical reference spectra of the remaining four structures using quantum-chemical calculations. Figure $2 \mathrm{~b}$ compares the predicted IR spectra (orange traces) to the experimental spectra of feature $\mathbf{A}$ and $\mathbf{B}$. All computed spectra show similarity to the experimental spectra, giving preliminary confirmation of the general chemical structure of feature $\mathbf{A}$ and $\mathbf{B}$. The frequencies of the two carbonyl stretching vibrations in the experimental spectra are very similar and correctly predicted only by the spectra of structure 1 and 2, suggesting that the $\mathrm{C}_{3} \mathrm{H}_{5} \mathrm{O}$-group is a 2-oxopropylgroup. Additionally, in the spectral region below $1500 \mathrm{~cm}^{-1}$, the experimental spectrum of feature $\mathbf{A}$ is overall best predicted by the computed spectrum of structure $\mathbf{1}$ and that of feature $\mathbf{B}$ by the spectrum computed for structure 2 . Note that the difference in peak shape in the $1200-1400 \mathrm{~cm}^{-1}$ region between the two features is relatively well explained by these two predicted spectra. In contrast, the experimentally observed bands around $1600 \mathrm{~cm}^{-1}$ of the two features are shifted relative to the predicted bands of structure $\mathbf{1}$ and $\mathbf{2}$. We attribute this discrepancy to the $\mathrm{NH}$-bending character of the vibration which, as noted above for P6C as well, often involves a strongly anharmonic behavior causing them to be shifted to higher wavenumbers in spectra predicted within the harmonic approximation. We note that the calculation does correctly predict the intensities and peak positions of this band in the two diastereomers relative to each other. Therefore, we tentatively identify feature $\mathbf{A}$ as (2S,6S)-6-(2oxopropyl)piperidine-2-carboxylic acid and feature $\mathbf{B}$ as its (2S,6R)-diastereomer (structure $\mathbf{1}$ and $\mathbf{2}$ in Figure 2).

Confirmation using IR spectra of synthetically obtained reference standards. Having made this tentative assignment, we proceeded to synthesize the reference standards for candidate structures $\mathbf{1}$ and $\mathbf{2}$. To this end, two synthetic routes were used which are shown in Scheme 2. As described in the supporting information, 6-hydroxynorleucine (7) was oxidized with chromium trioxide and subsequently reacted with acetoacetic acid to afford a mixture of diastereomers 1 and $\mathbf{2}^{40}$. Using LC in combination with an automated fraction collector we were able to obtain a fraction containing $\mathbf{1}$ only, which was used for IRIS experiments on $\mathbf{1}$ and its $\mathrm{m} / \mathrm{z}$ 128.0707 fragment. In addition, a novel route towards a mixture of $\mathbf{1}$ and $\mathbf{2}$ was developed starting from allysine ethylene acetal (8) to enable NMR characterisation (see Scheme 2). To this end, 8 was treated with acetoacetic acid under acidic conditions followed by benzylation to enable purification resulting in a mixture of diastereomers. Finally, deprotection of the mixture of diastereomers yielded $\mathbf{1}$ and $\mathbf{2}$ (0.7: 1.0, 1:2). At this stage, the relative stereochemistry could be assigned using NOESY NMR (See SI) ${ }^{41}$. To unambiguously assign the LC- retention time to one diastereoisomer, a stereoselective synthesis route was developed (see Scheme 2). Allysine ethylene acetal (8) was protected and acidic deprotection of the ethylidene acetal afforded enamine 9. Conversion of the enamine (9) into the $N, O$ acetal (10), reaction with the silyl enol ether of acetone and hydrogenolysis of the protecting groups afforded structure $2{ }^{41}$ This reference standard was used for IRIS experiments on 2 and its $m / z 128.0707$ fragment.

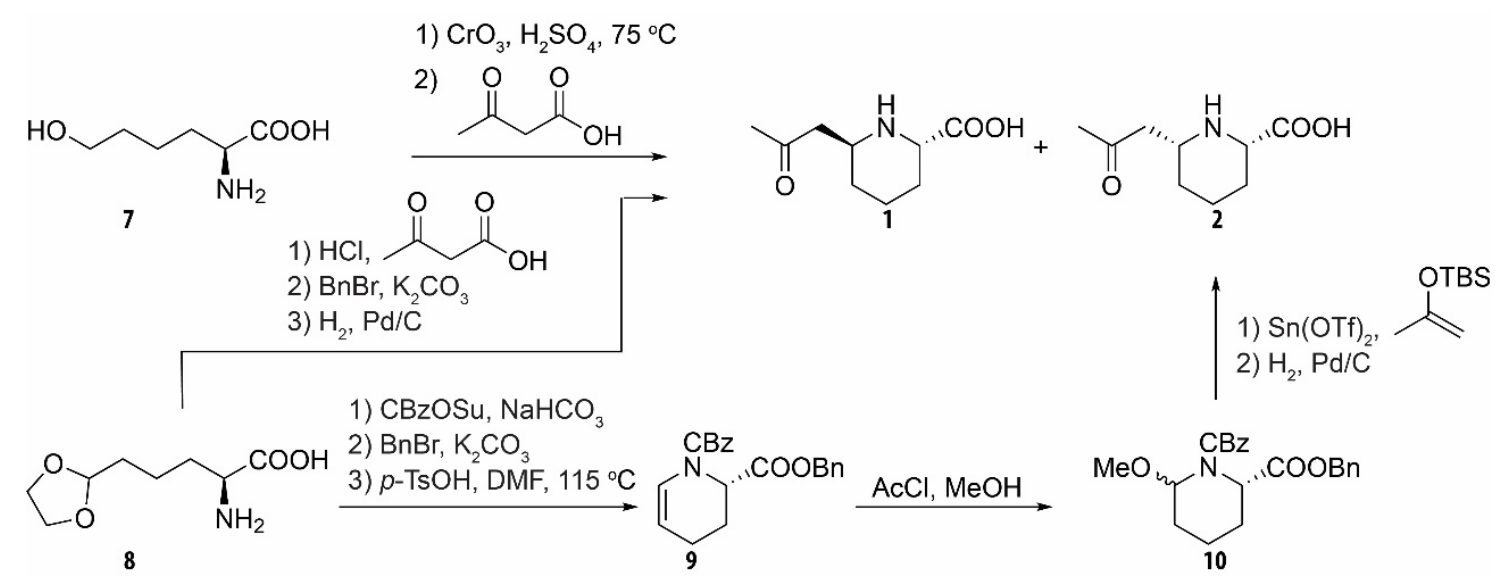

Scheme 2: Chemical syntheses of reference standards of structure $\mathbf{1}$ and $\mathbf{2}$ 
a

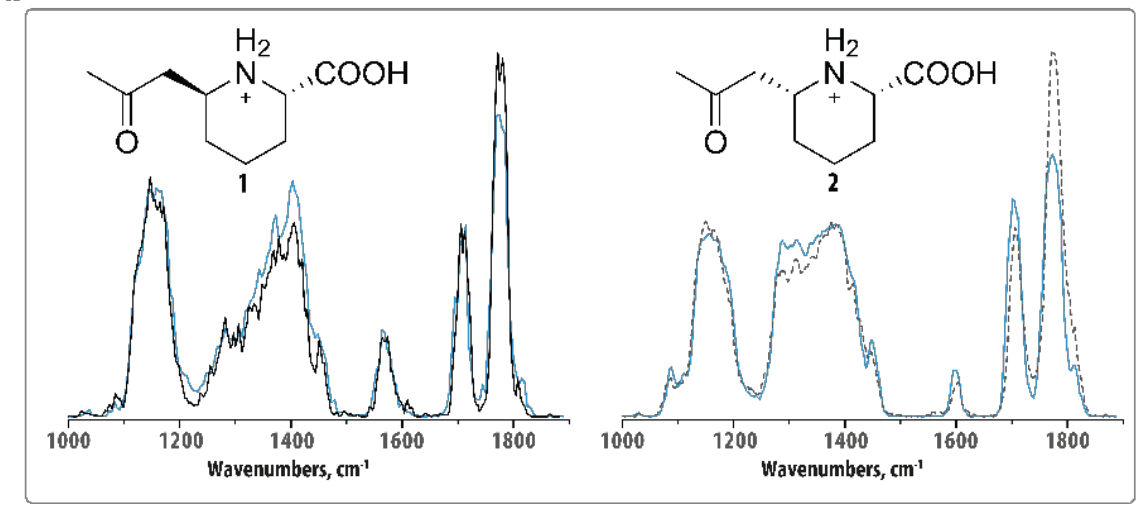

Figure 3 | Confirmation of the structural assignment of feature A and $\mathbf{B}$ with reference standards. (a) Comparison of the experimental IR spectra of feature A (black trace) and candidate structure $\mathbf{1}$ (blue trace, left panel) and the experimental IR spectra of feature $\mathbf{B}$ (dashed grey trace) and candidate structure $\mathbf{2}$ (blue trace, right panel). (b) Comparison of the experimental IR spectra of the $\mathrm{m} / \mathrm{z} 128.0707$ fragment of feature A (black trace) and structure $\mathbf{1}$ (blue trace). Molecular structures corresponding to the protonated reference standards are inlayed in each panel.

Figure 3a contains a comparison of the IR spectra of the protonated ions of the reference standards and the IR spectra of feature A (left panel) and B (right panel). Figure 4b shows the IR spectrum of the $\mathrm{m} / \mathrm{z} 128.0707$ fragment ion generated from structure 1 in comparison to the IR spectrum of the $\mathrm{m} / \mathrm{z}$ 128.0707 fragment generated from feature A. A similar comparison for the fragment of feature $\mathbf{B}$ and structure $\mathbf{2}$ is shown in Figure S3. All sets of spectra show an excellent match and each of the small deviations between the spectra can be attributed to minor fluctuations of experimental conditions, such as laser power, between experiments.

Finally, the reference standard of structure 2 was used to unequivocally confirm the assignment of the relative stereochemistry of feature $\mathbf{A}$ and $\mathbf{B}$. The connectivity and relative stereochemistry of $\mathbf{2}$ was assigned on the basis of ${ }^{1} \mathrm{H}$, ${ }^{13} \mathrm{C}$ and NOESY NMR experiments indicating the cis relationship between the substituents (see SI). With a pure standard of $\mathbf{2}$ in hand, its LC-MS retention time was measured which matched the retention time of feature $\mathbf{B}$.

As negative controls, we obtained reference standards for two closely related compounds, 1-(2-oxopropyl)piperidine-2carboxylic acid and 1-propanoylpiperidine-2-carboxylic acid containing a $\mathrm{C}_{3} \mathrm{H}_{5} \mathrm{O}$-substituent attached to the nitrogen of the piperidine. The spectral comparison in Figure S4 shows a clear mismatch between their IR spectra and the spectra of feature $\mathbf{A}$ and $\mathbf{B}$, further confirming the assignments above.

Additional biomarkers identified by IRIS. In addition to feature $\mathbf{A}$ and $\mathbf{B}$, two additional features, $\mathbf{C}$ and $\mathbf{D}$, were identified to have elevated levels in comparison to controls (though with a smaller fold change than feature $\mathbf{A}$ and $\mathbf{B}$ ). These $\mathrm{m} / \mathrm{z} 188.0919$ features have retention times of $1.45 \mathrm{~min}$ and 1.49 min and were not baseline separated. We therefore obtained an IR spectrum of a mixture of the co-eluting features $\mathbf{C}$ and $\mathbf{D}$ (black trace in Figure 4). Interestingly, the recorded IR spectrum shows a high-degree of similarity with the IR spectra of features A and B (see Figure 3b). Additionally, features $\mathbf{C}$ and $\mathbf{D}$ were also found to fragment to an $\mathrm{m} / \mathrm{z}$ 128.0707 (assigned as protonated P6C for feature A and B above). Taking this together, it suggests that feature $\mathbf{C}$ and $\mathbf{D}$ are the two diastereomers of 6-(carboxymethyl)piperidine-2carboxylic acid (structure $\mathbf{3}$ and $\mathbf{4}$ in Figure 4a). Figure 4a compares the experimental IR spectrum of the metabolite mixture with the calculated IR spectra of structure $\mathbf{3}$ and $\mathbf{4}$ (orange traces). We see that both theoretical spectra generally match the experimental IR spectrum of the mixture well. Overall, the IR spectrum of structure 3 (left panel of Figure 4a) seems to have a more significant contribution to the experimental spectrum, suggesting that it is present at a higher concentration than structure 4. Three features in the experimental IR spectrum directly suggest a contribution from structure 4: being the shoulder of the $\sim 1100 \mathrm{~cm}^{-1}$ peak, explained by the calculated band indicated by the red star, the intensity around $1300 \mathrm{~cm}^{-1}$, explained by the band indicated by the blue star, and the shoulder of the band around 1550 $\mathrm{cm}^{-1}$, explained by the calculated band indicated with the green star. Note that the latter is again redshifted compared to the calculation due to the anharmonic character of the $\mathrm{NH}$ bending vibration ${ }^{38-39}$.

We confirmed this assignment by developing a synthesis route yielding a reference standard of structure $\mathbf{4}$ (see Scheme 3 ). Briefly, 8 was reacted with malonic acid and the resulting products were benzylated to enable purification of the protected derivative 11. Final hydrogenolysis afforded structure 4 in a very low overall yield. NOESY NMR analysis indicated a cis-relationship of the substituents on the piperidine scaffold. We recorded an IR spectrum of structure 4 and Figure $4 \mathrm{~b}$ shows a comparison of this IR spectrum with the IR spectrum of the feature $\mathbf{C} / \mathbf{D}$ mixture. The three IR features predicted to originate from structure $\mathbf{4}$ are more prevalent in this IR spectrum (again indicated by the colour coded stars) and support its structural assignment.

Biochemical origins of biomarkers A-D. In a separate study, we focus on the implications of structure $\mathbf{1}$ and $\mathbf{2}$ on the pathophysiological understanding and diagnosis of PDEALDH7A ${ }^{1}$. In that study, we explore the (bio)chemical origins of structure $\mathbf{1}$ and $\mathbf{2}$ by conducting incubation experiments of P6C under physiological conditions. The synthesis of the reference standard described here showed that acetoacetic acid can react with P6C to afford $\mathbf{1}$ and 2. Acetoacetic acid is also known to be available in human body fluids. Indeed, incubation of P6C with acetoacetate under physiological conditions produced $\mathbf{1}$ and $\mathbf{2}$. We expect that the enol form of acetoacetic acid reacts with P6C and after decarboxylation affords feature $\mathbf{A}$ and $\mathbf{B}$. 
a

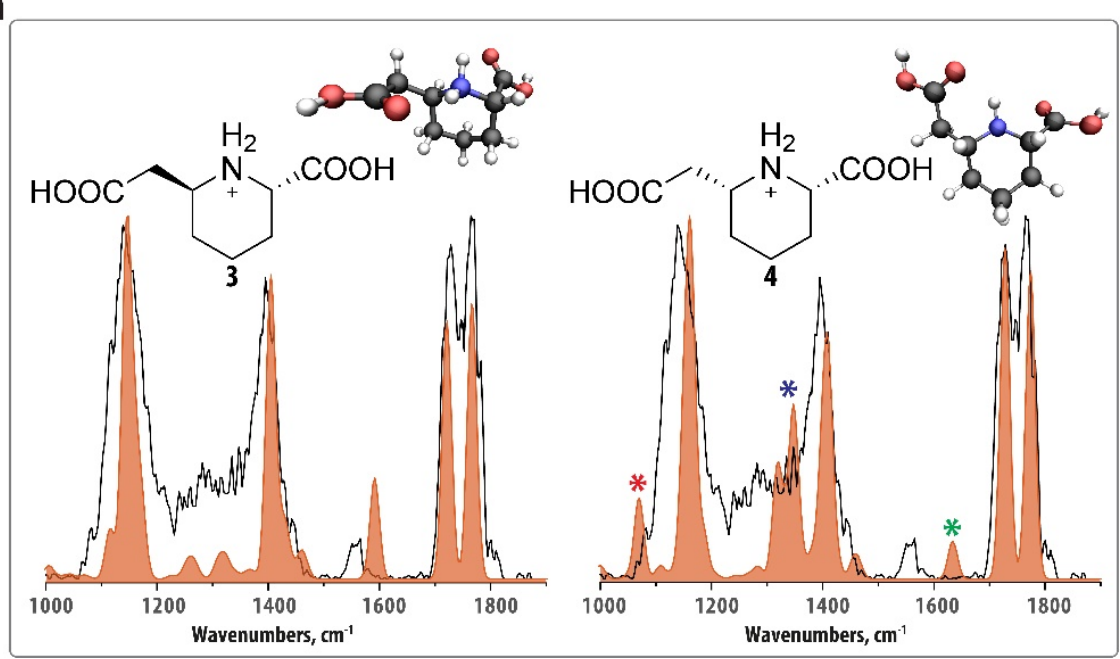

b

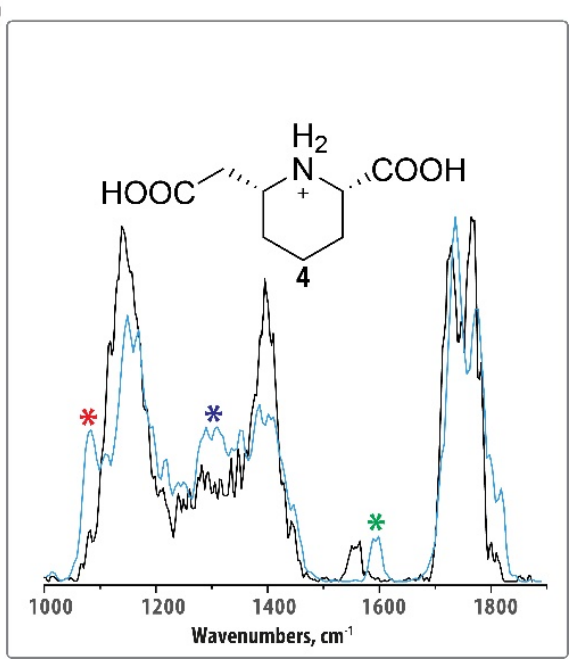

Figure 4 | Molecular identification of feature $\mathbf{C}$ and using computational modelling and IRIS. (a) Experimental IR spectrum of the co-eluting feature $\mathbf{C}$ and $\mathbf{D}$ (black trace in both panels) in comparison to the theoretical IR spectra of structure $\mathbf{3}$ and $\mathbf{4}$ (left and right panel, orange trace). Input structures for the computations and the conformations resulting from the quantum-chemical calculations are inlayed in each panel. Colored stars indicate bands that are particularly diagnostic for structure 4, see text. (b) Comparison of the experimental IR spectra of the co-eluting feature $\mathbf{C}$ and $\mathbf{D}$ (black trace) and protonated $\mathbf{4}$ (blue trace). The molecular structure of the protonated reference standard is inlayed in the panel.

Here, we hypothesize that malonic acid, which is similarly available in human body fluids, reacts with P6C to yield $\mathbf{3}$ and 4. We performed similar experiments by incubating malonic acid with P6C under physiological conditions in water, phosphate buffered saline (PBS), human control plasma and human control urine. The reaction of P6C with malonic acid occurred under all conditions tested and was confirmed by analysis with LC-MS and IRIS. Figure S5 shows the IR spectra of the observed features in all samples identifying them as a mixture of structure $\mathbf{3}$ and $\mathbf{4}$. These observations point in a more general sense to the fact that P6C reacts as an imine with any carbonyl containing species that can form stable enol tautomers, such as the $\beta$-dicarbonyl compounds acetoacetate and malonate. In contrast, P6C reacts in its enamine tautomeric form in a cross coupling with the aldehyde of PLP, leading to the secondary pyridoxine deficiency in PDE-ALDH7A1.

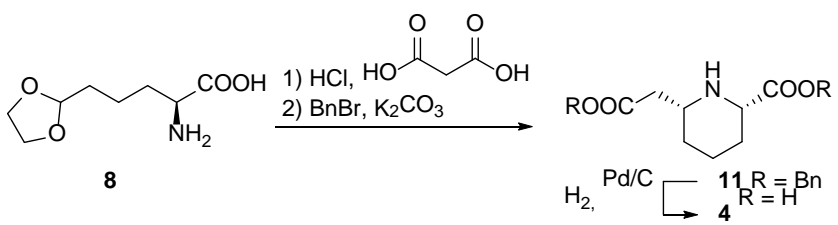

Scheme 3: Chemical synthesis of a reference standard of structure 4.

\section{CONCLUSIONS}

Using our NGMS/IRIS pipeline, we have now shown that the molecular structures of unknown $\mathrm{m} / \mathrm{z}$-features detected in untargeted metabolomics workflows can be annotated using IRIS, theoretically predicted IR spectra and synthesized reference compounds. Features are preliminarily annotated by comparison of their experimentally measured IR spectra with theoretically predicted IR spectra generated for candidate molecular structures. Furthermore, the most relevant candidate structures necessary to consider in this approach can often be determined directly from structural information (functional group vibrational assignments) contained in the IR spectra of the unknowns. Importantly, this approach dramatically limits the scope of synthetic standards required for final identification. Here, we have applied this technique to determine the molecular structures of four previously unknown biomarkers for PDE-ALDH7A1. We have identified them as two sets of diastereomeric derivatives of P6C, a known metabolite in the degradation of L-lysine that accumulates in patients with PDE-ALDH7A1. This logically connects these metabolites to known biochemical routes of Llysine metabolism, but also suggests a disease-specific expansion of this metabolic pathway in PDE-ALDH7A1. The identification of the molecular structures of the biomarkers allows us to deduce their likely biochemical origins, which may lead to a more complete understanding of the chemical basis for the pathophysiology of PDE-ALDH7A1 and to new treatment targets. 


\section{METHODS}

Sample preparation. Plasma, CSF and urine samples were stored at $-80{ }^{\circ} \mathrm{C}$ and thawed at $4{ }^{\circ} \mathrm{C}$ just before sample preparation. $100 \mu$ of sample was combined with $400 \mu$ lice-cold methanol/ethanol 50:50 [v/v] and mixed for $15 \mathrm{~s}$ using a vortex mixer. For the samples used in metabolic profiling, the methanol/ethanol mixture contained five internal standards (caffeine- $d_{3} 0.88 \mu \mathrm{mol} / \mathrm{L}$, hippuric- $d_{5}$ acid $0.22 \mu \mathrm{mol} / \mathrm{L}$, nicotinic- $d_{4}$ acid 0.88 $\mu \mathrm{mol} / \mathrm{L}$, octanoyl-L-carnitine- $d_{3} 0.22 \mu \mathrm{mol} / \mathrm{L}$, L-phenyl- $d_{5}-$ alanine $0.44 \mu \mathrm{mol} / \mathrm{L}$ (all from $\mathrm{C} / \mathrm{D} / \mathrm{N}$ Isotopes, PointeClaire, Canada)). The resulting mixture was incubated for $20 \mathrm{~min}$ at $4{ }^{\circ} \mathrm{C}$ and centrifuged for $15 \mathrm{~min}$ at $4{ }^{\circ} \mathrm{C}(18600$ g). The supernatant (350 $\mu \mathrm{L})$ was dried in a centrifugal vacuum evaporator (Eppendorf). The dried sample was reconstituted in $100 \mathrm{~mL}$ deionised water/methanol 90:10 [v/v] with $0.1 \%$ formic acid, mixed for $15 \mathrm{~s}$ with a vortex mixer at room temperature and centrifuged for $15 \mathrm{~min}$ at 18600 g. The supernatant (90 $\mu \mathrm{L})$ was used for LC-MS analysis.

Metabolic profiling. A full description of the untargeted metabolic screening procedure and statistical analysis leading to the detection of the biomarkers discussed in this work are described separately ${ }^{1}$. In short, LC-MS runs were preformed using an Agilent 1290 ultra-high pressure LC system coupled to a 6545 QTOF mass spectrometer. Data acquisition and analysis was done using Agilent Mass Hunter (version B.08.00). Separations were performed using a flow rate of $0.4 \mathrm{~mL} / \mathrm{min}$ on a Waters Acquity HSS T3 C18 column $(100 \times 2.1 \mathrm{~mm}$ i.d., $1.8 \mu \mathrm{m}$ particles, $100 \AA$ pore size) held at $40{ }^{\circ} \mathrm{C}$. The mobile phase consisted of $0.1 \%(\mathrm{v} / \mathrm{v})$ formic acid in water and $0.1 \%(\mathrm{v} / \mathrm{v})$ formic acid in 99:1 (v/v) methanol: water (mobile phase B). After a hold at $99 \%$ A for 1 minute, a gradient of 15 minutes was run to $100 \% \mathrm{~B}$, followed by a hold of 4 minutes at $100 \% \mathrm{~B}$ and a return to $99 \%$ A of 1 minute. An equilibration time of 4 minutes was used leading to a total analysis time of 25 minutes. Injection volumes of $2 \mu \mathrm{L}$ were used for all samples. Alignment and feature extraction was performed using the open access software package $\mathrm{XCMS}^{42}$ and twosided t-tests were performed to identify significantly altered features between patients and controls. See Coene et al. $^{30}$ and Engelke et al. ${ }^{1}$ for details regarding this procedure.

Infrared ion spectroscopy. IRIS experiments were performed in a quadrupole ion trap mass spectrometer (Bruker, AmaZon Speed ETD) that has been modified to provide optical access to the stored ions for spectroscopy experiments. Details of these modifications and operation of the experiment are described elsewhere ${ }^{43}$. Fractions containing metabolic features of interest were collected with a Bruker Elute SP HPLC system and a two-position 6-port switching valve controlled by the quadrupole ion trap. The separations were performed using the same separation procedure as described above but with a mobile phase consisting of $10 \mathrm{mM}$ acetic acid in water (mobile phase $\mathrm{A}$ ) and $10 \mathrm{mM}$ acetic acid in methanol (mobile phase $\mathrm{B})$. For feature $\mathbf{A}$ and $\mathbf{B}$, the switching valve was used to divert the eluent containing the features to Eppendorf tubes, which were analysed by IRIS in a separate experiment by infusing the fractions with a syringe pump $(120-180 \mu \mathrm{L} / \mathrm{h})$. For feature $\mathbf{C}$ and $\mathbf{D}$, the LC and IRIS experiment were combined by installing an $80 \mu \mathrm{L}$ sample loop between the switching valve and the ion source of the ion trap. As soon as the LC peak of interest arrived at the mass spectrometer the valve was switched, such that the remainder of the feature material was infused by a syringe pump (120 $\mu \mathrm{L} / \mathrm{h})$ for IRIS experiments. For reference IR measurements, 1-propanoylpiperidine-2-carboxylic acid (Enamine Ltd. (Kyiv, Ukraine)) and 1-(2oxopropyl)piperidine-2-carboxylic acid (see supporting info for the synthesis procedure), compound $\mathbf{2}$ and compound 4 were dissolved in 50:50 (v/v) acetonitrile:water $\left(\sim 10^{-7} \mathrm{M}\right)$ and infused directly with the syringe pump for IRIS experiments $(180 \mu \mathrm{L} / \mathrm{h})$. Compound $\mathbf{1}$ was isolated from a mixture of $\mathbf{1}$ and $\mathbf{2}$ by performing the chromatographic separation detailed above and collecting the fraction of the eluent containing 1 using a Foxy R2 fraction collector equipped with two 96-well plates. This fraction was infused with the syringe pump for IRIS experiments at $120-180 \mu \mathrm{L} / \mathrm{h}$ flow rates.

To obtain IRIS spectra, features of interest were massisolated and stored for $200 \mathrm{~ms}$ in the quadrupole ion trap. During this time, they were irradiated using the FELIX infrared free electron laser ${ }^{44}$, which produced IR radiation in the form of $\sim 10 \mu$ s macropulses of $30-200 \mathrm{~mJ}$ at a 10 $\mathrm{Hz}$ repetition rate (bandwidth $\sim 0.4 \%$ of the centre frequency). IR spectra were obtained by scanning the laser with $3 \mathrm{~cm}^{-1}$ steps and recording a mass spectrum after irradiation at each frequency step. MS spectra were recorded as an average of $4-8$ scans. When the laser frequency is resonant with a molecular vibration of the trapped ions, absorption takes place which increases the internal energy of the stored ions leading, after the absorption of multiple photons, to unimolecular dissociation. The amount of IR absorption is measured by the degree of fragmentation at each wavelength point by relating the precursor and fragment ion intensities (yield = $\Sigma$ (fragment ions) $/(\Sigma$ (parent + fragment ions)). The laser frequency was calibrated using a grating spectrometer and the fragmentation yield was linearly corrected for frequency-dependent pulse energy variation. IR spectra of fragment ions were obtained by first using collision induced dissociation (50 ms, fragmentation amplitude optimized on fragment ion signal) on the relevant precursor ion before mass isolation of the desired fragment ion and IR laser irradiation as described above.

H/D exchange. In order to perform $H / D$ exchange on feature $\mathbf{A}$ and $\mathbf{B}$ an LC-fraction containing both unknown analytes was collected according to the procedure described above. The fraction was dried in a centrifugal vacuum evaporator (Eppendorf) and reconstituted in 100 $\mu \mathrm{L}$ deuterated methanol. Subsequently, the H/Dexchanged fraction was infused at $180 \mu \mathrm{L} / \mathrm{h}$ to the quadrupole ion trap mass spectrometer. It was observed that the majority $(>70 \%)$ of the ions corresponding to feature $\mathbf{A}$ and $\mathbf{B}$ had undergone three exchanges. The 
remaining part of the ion population was observed at $\mathrm{m} / \mathrm{z}$ 188 , which corresponds to two exchanges. It is well-known that the trapping region of the ion trap used in these experiments is not completely water-free. Therefore, the observation of ions with $\mathrm{m} / \mathrm{z} 188$ likely due to backexchange inside the mass-spectrometer.

Quantum chemical calculations. To obtain predicted IR spectra, density functional theory (DFT) calculations were performed using the Gaussian16 software package ${ }^{45}$. For each ion of interest several molecular structures were manually defined based on chemical intuition and known gas-phase ion chemistry. Structures were optimised at the B3LYP/6-31++G(d,p) level of theory followed by a vibrational frequency calculation within the harmonic approximation. Calculated spectra were scaled using a linear scaling factor of 0.975 and broadened using a Gaussian line shape function $\left(20 \mathrm{~cm}^{-1}\right.$ full width at half maximum) to facilitate comparison with the experimental IR spectra.

Data Availability. All data are included in the manuscript and the SI. Raw research data is available upon request from the corresponding authors.

\section{REFERENCES}

1. Engelke, U. F. H.; van Outersterp, R.; Merx, J.; van Geenen, F. A. M. G.; van Rooij, A.; Berden, G.; Huigen, M. C. D. G.; Kluijtmans, L. A. J.; Peters, T. M. A.; Al-Shekaili, H. H.; Leavitt, B. R.; de Vrieze, E.; Broekman, S.; van Wijk, E.; Tseng, L. A.; Kulkarni, P.; Rutjes, F. P. J. T.; Mecinović, J.; Struys, E. A.; Jansen, L. A.; Gospe, S. M.; Mercimek-Andrews, S.; Hyland, K.; Willemsen, M. A. A. P.; Bok, L. A.; van Karnebeek, C. D. M.; Wevers, R. A.; Boltje, T. J.; Oomens, J.; Martens, J.; Coene, K. L. M., Identification of novel biomarkers for pyridoxinedependent epilepsy using untargeted metabolomics and infrared ion spectroscopy biochemical insights and clinical implications. medRxiv 2021, 2021.01.22.20248925.

2. Dunn, W. B.; Erban, A.; Weber, R. J. M.; Creek, D. J.; Brown, M.; Breitling, R.; Hankemeier, T.; Goodacre, R.; Neumann, S.; Kopka, J.; Viant, M. R., Mass appeal: metabolite identification in mass spectrometry-focused untargeted metabolomics. Metabolomics 2013, 9 (1), 44-66.

3. Hufsky, F.; Böcker, S., Mining molecular structure databases: Identification of small molecules based on fragmentation mass

\section{Acknowledgments}

The authors gratefully acknowledge the excellent technical assistance from the FELIX group and of Siebolt de Boer, Ed van der Heeft and Joris Reintjes from the Translational Metabolic Laboratory. We thank the Nederlandse Organisatie voor Wetenschappelijk Onderzoek (NWO) for the support of the FELIX Laboratory. The authors are appreciative of the friendly and helpful support provided by Bruker related to hardware and software modifications of our mass spectrometers, in particular Dr. Christoph Gebhardt. Financial support for this project was provided by NWO Chemical Sciences under projects NWO-TTW nr. 15769 and TKI-LIFT nr. 731.017.419 awarded to JO. This work was also supported by an ERC-Stg awarded to TJB (GlycoEdit, 758913). We thank NWO Exact and Natural Sciences and the SURFsara Supercomputer Centre for providing the computational time and resources (grant 16327). Parts of this work were financially supported by an Interfacultary Collaboration Grant from Radboud University (awarded to KLMC and JM). This research made use of metabolomics infrastructure that is part of the NWO-funded Netherlands X-omics initiative, project 184.034.019.

spectrometry data. Mass Spectrometry Reviews 2017, 36 (5), 624-633.

4. Cui, L.; Lu, H.; Lee, Y. H., Challenges and emergent solutions for LC-MS/MS based untargeted metabolomics in diseases. Mass Spectrometry Reviews 2018, 37 (6), 772-792.

5. Hufsky, F.; Scheubert, K.; Böcker, S., Computational mass spectrometry for smallmolecule fragmentation. TrAC Trends in Analytical Chemistry 2014, 53, 41-48.

6. van Outersterp, R. E.; Houthuijs, K. J.; Berden, G.; Engelke, U. F.; Kluijtmans, L. A.; Wevers, R. A.; Coene, K. L.; Oomens, J.; Martens, J., Reference-standard free metabolite identification using infrared ion spectroscopy. International Journal of Mass Spectrometry 2019, 443, 77-85.

7. Martens, J.; Berden, G.; Bentlage, H.; Coene, K. L. M.; Engelke, U. F.; Wishart, D.; van Scherpenzeel, M.; Kluijtmans, L. A. J.; Wevers, R. A.; Oomens, J., Unraveling the unknown areas of the human metabolome: the role of infrared ion spectroscopy. Journal of Inherited Metabolic Disease 2018, 41, 367-377.

8. Martens, J.; Koppen, V.; Berden, G.; Cuyckens, F.; Oomens, J., Combined Liquid Chromatography-Infrared Ion Spectroscopy for Identification of Regioisomeric Drug Metabolites. Analytical Chemistry 2017, 89 (8), 4359-4362. 
9. Martens, J.; Berden, G.; van Outersterp, R. E.; Kluijtmans, L. A. J.; Engelke, U. F.; van Karnebeek, C. D. M.; Wevers, R. A.; Oomens, J., Molecular identification in metabolomics using infrared ion spectroscopy. Scientific Reports 2017, 7 (1), 3363.

10. Cismesia, A. P.; Bell, M. R.; Tesler, L. F.; Alves, M.; Polfer, N. C., Infrared ion spectroscopy: an analytical tool for the study of metabolites. Analyst 2018, 143 (7), 1615-1623.

11. Gorlova, O.; Colvin, S. M.; Brathwaite, A.; Menges, F. S.; Craig, S. M.; Miller, S. J.; Johnson, M. A., Identification and Partial Structural Characterization of Mass Isolated Valsartan and Its Metabolite with Messenger Tagging Vibrational Spectroscopy. Journal of the American Society for Mass Spectrometry 2017, 28 (11), 2414-2422.

12. Cismesia, A. P.; Bailey, L. S.; Bell, M. R.; Tesler, L. F.; Polfer, N. C., Making Mass Spectrometry See the Light: The Promises and Challenges of Cryogenic Infrared Ion Spectroscopy as a Bioanalytical Technique. Journal of the American Society for Mass Spectrometry 2016, 27 (5), 757-766.

13. Schindler, B.; Laloy-Borgna, G.; Barnes, L.; Allouche, A.-R.; Bouju, E.; Dugas, V.; Demesmay, C.; Compagnon, I., Online Separation and Identification of Isomers Using Infrared Multiple Photon Dissociation Ion Spectroscopy Coupled to Liquid Chromatography: Application to the Analysis of Disaccharides Regio-Isomers and Monosaccharide Anomers. Analytical Chemistry 2018, 90 (20), 11741-11745.

14. Corinti, D.; Maccelli, A.; Crestoni, M. E.; Cesa, S.; Quaglio, D.; Botta, B.; Ingallina, C.; Mannina, L.; Tintaru, A.; Chiavarino, B.; Fornarini, S., IR ion spectroscopy in a combined approach with MS/MS and IM-MS to discriminate epimeric anthocyanin glycosides (cyanidin 3-Oglucoside and -galactoside). International Journal of Mass Spectrometry 2019, 444, 116179.

15. Walhout, E. Q.; Dorn, S. E.; Martens, J.; Berden, G.; Oomens, J.; Cheong, P. H. Y.; Kroll, J. H.; O’Brien, R. E., Infrared Ion Spectroscopy of Environmental Organic Mixtures: Probing the Composition of $\alpha$-Pinene Secondary Organic Aerosol. Environmental Science \& Technology 2019, 53 (13), 7604-7612.
16. Lagatie, O.; Verheyen, A.; Van Asten, S.; Odiere, M. R.; Djuardi, Y.; Levecke, B.; Vlaminck, J.; Mekonnen, Z.; Dana, D.; T’Kindt, R.; Sandra, K.; van Outersterp, R.; Oomens, J.; Lin, R.; Dillen, L.; Vreeken, R.; Cuyckens, F.; Stuyver, L. J., 2-Methyl-pentanoyl-carnitine (2MPC): a urine biomarker for patent Ascaris lumbricoides infection. Scientific Reports 2020, 10 (1), 15780.

17. van Outersterp, R. E.; Moons, S. J.; Engelke, U. F. H.; Bentlage, H.; Peters, T. M. A.; van Rooij, A.; Huigen, M. C. D. G.; Boer, S.; van der Heeft, E.; Kluijtmans, L. A. J.; van Karnebeek, C. D. M.; Wevers, R. A.; Berden, G.; Oomens, J.; Boltje, T. J.; Coene, K. L. M.; Martens, J., Amadori rearrangement products as potential biomarkers for inborn errors of amino acid metabolism. Communications biology.

18. Martens, J.; van Outersterp, R. E.; Vreeken, R. J.; Cuyckens, F.; Coene, K. L. M.; Engelke, U. F.; Kluijtmans, L. A. J.; Wevers, R. A.; Buydens, L. M. C.; Redlich, B.; Berden, G.; Oomens, J., Infrared ion spectroscopy: New opportunities for small-molecule identification in mass spectrometry - A tutorial perspective. Analytica Chimica Acta 2020, 1093, 1-15.

19. Struys, E. A.; Jakobs, C., Metabolism of lysine in $\alpha$-aminoadipic semialdehyde dehydrogenase-deficient fibroblasts: Evidence for an alternative pathway of pipecolic acid formation. FEBS Letters 2010, 584 (1), 181-186.

20. Crowther, L. M.; Mathis, D.; Poms, M.; Plecko, B., New insights into human lysine degradation pathways with relevance to pyridoxine-dependent epilepsy due to antiquitin deficiency. Journal of Inherited Metabolic Disease 2019, 42 (4), 620-628.

21. Mills, P. B.; Struys, E.; Jakobs, C.; Plecko, B.; Baxter, P.; Baumgartner, M.; Willemsen, M. A. A. P.; Omran, H.; Tacke, U.; Uhlenberg, B.; Weschke, B.; Clayton, P. T., Mutations in antiquitin in individuals with pyridoxinedependent seizures. Nature Medicine 2006, 12 (3), 307-309.

22. Takazawa, O.; Kogami, K.; Hayashi, K., New Crossed Aldol Reaction. Reaction of Enamines with Aldehydes Activated by Lewis Acids. Bulletin of the Chemical Society of Japan 1985, 58 (8), 2427-2428. 
23. Babler, J. H.; Atwood, M. C.; Freaney, J. E.; Viszlay, A. R., The role of imine-enamine tautomerism in effecting cross-aldol condensations. Tetrahedron Letters 2007, 48 (43), 7665-7667.

24. Coughlin, C. R.; Swanson, M. A.; Spector, E.; Meeks, N. J. L.; Kronquist, K. E.; Aslamy, M.; Wempe, M. F.; van Karnebeek, C. D. M.; Gospe, S. M.; Aziz, V. G.; Tsai, B. P.; Gao, H.; Nagy, P. L.; Hyland, K.; van Dooren, S. J. M.; Salomons, G. S.; Van Hove, J. L. K., The genotypic spectrum of ALDH7A1 mutations resulting in pyridoxine dependent epilepsy: a common epileptic encephalopathy. Journal of Inherited Metabolic Disease 2018.

25. Struys, E. A.; Bok, L. A.; Emal, D.; Houterman, S.; Willemsen, M. A.; Jakobs, C., The measurement of urinary $\Delta 1$-piperideine-6carboxylate, the alter ego of $\alpha$-aminoadipic semialdehyde, in Antiquitin deficiency. Journal of Inherited Metabolic Disease 2012, 35 (5), 909916.

26. Yuzyuk, T.; Liu, A.; Thomas, A.; Wilson, J. E.; De Biase, I.; Longo, N.; Pasquali, M., A novel method for simultaneous quantification of alpha-aminoadipic semialdehyde/piperideine-6carboxylate and pipecolic acid in plasma and urine. Journal of Chromatography B 2016, 10171018, 145-152.

27. Xue, J.; Wang, J.; Gong, P.; Wu, M.; Yang, W.; Jiang, S.; Wu, Y.; Jiang, Y.; Zhang, Y.; Yuzyuk, T.; Li, H.; Yang, Z., Simultaneous quantification of alpha-aminoadipic semialdehyde, piperideine-6-carboxylate, pipecolic acid and alpha-aminoadipic acid in pyridoxine-dependent epilepsy. Scientific Reports 2019, 9 (1), 11371.

28. Mathew, E. M.; Moorkoth, S.; Lewis, L.; Rao, P., Biomarker Profiling for Pyridoxine Dependent Epilepsy in Dried Blood Spots by HILIC-ESI-MS. International Journal of Analytical Chemistry 2018, 2018, 2583215.

29. Jung, S.; Tran, N.-T. B.; Gospe, S. M.; Hahn, S. H., Preliminary investigation of the use of newborn dried blood spots for screening pyridoxine-dependent epilepsy by LC-MS/MS. Molecular Genetics and Metabolism 2013, 110 (3), 237-240.

30. Coene, K. L. M.; Kluijtmans, L. A. J.; van der Heeft, E.; Engelke, U. F. H.; de Boer, S.;
Hoegen, B.; Kwast, H. J. T.; van de Vorst, M.; Huigen, M. C. D. G.; Keularts, I. M. L. W.; Schreuder, M. F.; van Karnebeek, C. D. M.; Wortmann, S. B.; de Vries, M. C.; Janssen, M. C. H.; Gilissen, C.; Engel, J.; Wevers, R. A., Nextgeneration metabolic screening: targeted and untargeted metabolomics for the diagnosis of inborn errors of metabolism in individual patients. Journal of Inherited Metabolic Disease 2018, 41 (3), 337-353.

31. van Karnebeek, C. D. M.; Tiebout, S. A.; Niermeijer, J.; Poll-The, B. T.; Ghani, A.; Coughlin Ii, C. R.; Van Hove, J. L. K.; Richter, J. W.; Christen, H. J.; Gallagher, R.; Hartmann, H.; Stockler-Ipsiroglu, S., Pyridoxine-Dependent Epilepsy: An Expanding Clinical Spectrum. Pediatric Neurology 2016, 59, 6-12.

32. Wishart, D. S.; Tzur, D.; Knox, C.; Eisner, R.; Guo, A. C.; Young, N.; Cheng, D.; Jewell, K.; Arndt, D.; Sawhney, S.; Fung, C.; Nikolai, L.; Lewis, M.; Coutouly, M.-A.; Forsythe, I.; Tang, P.; Shrivastava, S.; Jeroncic, K.; Stothard, P.; Amegbey, G.; Block, D.; Hau, D. D.; Wagner, J.; Miniaci, J.; Clements, M.; Gebremedhin, M.; Guo, N.; Zhang, Y.; Duggan, G. E.; MacInnis, G. D.; Weljie, A. M.; Dowlatabadi, R.; Bamforth, F.; Clive, D.; Greiner, R.; Li, L.; Marrie, T.; Sykes, B. D.; Vogel, H. J.; Querengesser, L., HMDB: the Human Metabolome Database. Nucleic Acids Res. 2007, 35 (suppl_1), D521-D526.

33. Wishart, D. S.; Knox, C.; Guo, A. C.; Eisner, R.; Young, N.; Gautam, B.; Hau, D. D.; Psychogios, N.; Dong, E.; Bouatra, S.; Mandal, R.; Sinelnikov, I.; Xia, J.; Jia, L.; Cruz, J. A.; Lim, E.; Sobsey, C. A.; Shrivastava, S.; Huang, P.; Liu, P.; Fang, L.; Peng, J.; Fradette, R.; Cheng, D.; Tzur, D.; Clements, M.; Lewis, A.; De Souza, A.; Zuniga, A.; Dawe, M.; Xiong, Y.; Clive, D.; Greiner, R.; Nazyrova, A.; Shaykhutdinov, R.; Li, L.; Vogel, H. J.; Forsythe, I., HMDB: a knowledgebase for the human metabolome. Nucleic Acids Res. 2009, 37 (suppl_1), D603D610.

34. Wishart, D. S.; Jewison, T.; Guo, A. C.; Wilson, M.; Knox, C.; Liu, Y.; Djoumbou, Y.; Mandal, R.; Aziat, F.; Dong, E.; Bouatra, S.; Sinelnikov, I.; Arndt, D.; Xia, J.; Liu, P.; Yallou, F.; Bjorndahl, T.; Perez-Pineiro, R.; Eisner, R.; Allen, F.; Neveu, V.; Greiner, R.; Scalbert, A., HMDB 3.0-The Human Metabolome Database 
in 2013. Nucleic Acids Res. 2013, 41 (D1), D801D807.

35. Wishart, D. S.; Feunang, Y. D.; Marcu, A.; Guo, A. C.; Liang, K.; Vázquez-Fresno, R.; Sajed, T.; Johnson, D.; Li, C.; Karu, N.; Sayeeda, Z.; Lo, E.; Assempour, N.; Berjanskii, M.; Singhal, S.; Arndt, D.; Liang, Y.; Badran, H.; Grant, J.; SerraCayuela, A.; Liu, Y.; Mandal, R.; Neveu, V.; Pon, A.; Knox, C.; Wilson, M.; Manach, C.; Scalbert, A., HMDB 4.0: the human metabolome database for 2018. Nucleic Acids Res. 2018, 46 (D1), D608D617.

36. Smith, C. A.; Maille, G. O.; Want, E. J.; Qin, C.; Trauger, S. A.; Brandon, T. R.; Custodio, D. E.; Abagyan, R.; Siuzdak, G., METLIN: A Metabolite Mass Spectral Database. Therapeutic Drug Monitoring 2005, 27 (6).

37. Pence, H. E.; Williams, A., ChemSpider: An Online Chemical Information Resource. Journal of Chemical Education 2010, 87 (11), 1123-1124.

38. Boles, G. C.; Hightower, R. L.; Berden, G.; Oomens, J.; Armentrout, P. B., Zinc and Cadmium Complexation of l-Threonine: An Infrared Multiple Photon Dissociation Spectroscopy and Theoretical Study. The Journal of Physical Chemistry B 2019, 123 (44), 9343-9354.

39. Coates, R. A.; McNary, C. P.; Boles, G. C.; Berden, G.; Oomens, J.; Armentrout, P. B., Structural characterization of gas-phase cysteine and cysteine methyl ester complexes with zinc and cadmium dications by infrared multiple photon dissociation spectroscopy. Physical Chemistry Chemical Physics 2015, 17 (39), 25799-25808.

40. Hasse, K.; Hess, J.; Hörnig, H. W., Darstellung und Verhalten von 1.5-Didehydronorhygrin und 1.6-Didehydro-isopelletierin. Chemische Berichte 1971, 104 (8), 2420-2426.

41. Botman, P. N. M.; Dommerholt, F. J.; de Gelder, R.; Broxterman, Q. B.; Schoemaker, H. E.; Rutjes, F. P. J. T.; Blaauw, R. H., Diastereoselective Synthesis of (2S,5R)-5Hydroxypipecolic Acid and 6-Substituted Derivatives. Organic Letters 2004, 6 (26), 49414944.

42. Tautenhahn, R.; Patti, G. J.; Rinehart, D.; Siuzdak, G., XCMS Online: A Web-Based Platform to Process Untargeted Metabolomic Data. Analytical Chemistry 2012, 84 (11), 50355039.
43. Martens, J.; Berden, G.; Gebhardt, C. R.; Oomens, J., Infrared ion spectroscopy in a modified quadrupole ion trap mass spectrometer at the FELIX free electron laser laboratory. Review of Scientific Instruments 2016, 87 (10), 103108.

44. Oepts, D.; van der Meer, A. F. G.; van Amersfoort, P. W., The Free-Electron-Laser user facility FELIX. Infrared Physics \& Technology 1995, 36 (1), 297-308.

45. Frisch, M. J.; Trucks, G. W.; Schlegel, H. B.; G. E. Scuseria; Robb, M. A.; Cheeseman, J. R.; Scalmani, G.; Barone, V.; Petersson, G. A.; Nakatsuji, H.; Li, X.; Caricato, M.; Marenich, A. V.; Bloino, J.; Janesko, B. G.; Gomperts, R.; Mennucci, B.; Hratchian, H. P.; Ortiz, J. V.; Izmaylov, A. F.; Sonnenberg, J. L.; WilliamsYoung, D.; Ding, F.; Lipparini, F.; Egidi, F.; Goings, J.; Peng, B.; Petrone, A.; Henderson, T.; Ranasinghe, D.; Zakrzewski, V. G.; Gao, J.; Rega, N.; Zheng, G.; Liang, W.; Hada, M.; Ehara, M.; K. Toyota; Fukuda, R.; Hasegawa, J.; M. Ishida; Nakajima, T.; Y. Honda; Kitao, O.; H. Nakai; T. Vreven; Throssell, K.; Jr., J. A. M.; J. E. Peralta; F. Ogliaro; M. J. Bearpark; Heyd, J. J.; Brothers, E. N.; Kudin, K. N.; Staroverov, V. N.; Keith, T. A.; Kobayashi, R.; Normand, J.; Raghavachari, K.; Rendell, A. P.; Burant, J. C.; Iyengar, S. S.; Tomasi, J.; Cossi, M.; Millam, J. M.; Klene, M.; Adamo, C.; Cammi, R.; Ochterski, J. W.; Martin, R. L.; Morokuma, K.; Farkas, O.; Foresman, J. B.; Fox, D. J., Gaussian 16, Revision A.03. Gaussian, Inc., Wallingford CT 2016. 